\title{
Far-Field Reconstruction from Transient Near-Field Measurement Using Cylindrical Modal Development
}

\author{
R. Rammal, ${ }^{1}$ M. Lalande, ${ }^{1}$ E. Martinod, ${ }^{1}$ N. Feix, ${ }^{1}$ M. Jouvet, ${ }^{1}$ J. Andrieu, ${ }^{1}$ and B. Jecko ${ }^{2}$ \\ ${ }^{1}$ XLIM, UMR CNRS No. 6172, 7 rue Jules Vallès, 19100 Brive, France \\ ${ }^{2}$ XLIM, UMR CNRS No. 6172, 123 rue Albert Thomas, 87000 Limoges, France
}

Correspondence should be addressed to R. Rammal, rabia.rammal@xlim.fr

Received 18 May 2009; Revised 30 August 2009; Accepted 31 October 2009

Recommended by Miguel Ferrando

The aim of this work is to get far field radiation patterns for any radiating source from transient acquisition, in a large frequency range. An outdoor transient Ultra-Wideband near-field measurement base will be installed, a single time pulse radiated by the source will cover the desired spectrum, and the accurate determination of far field radiations will be accomplished by means of cylindrical waves' modal development. This method uses simplified test equipments, easy to be installed, and it reduces measurement costs.

Copyright (C) 2009 R. Rammal et al. This is an open access article distributed under the Creative Commons Attribution License, which permits unrestricted use, distribution, and reproduction in any medium, provided the original work is properly cited.

\section{Introduction}

Interested in measuring radiations of antennas placed in their context of use, such as antennas placed inside and outside a vehicle, or radiations of electronic systems integrated in many functional parts of a vehicle, the near field (NF) to far field (FF) transformation technique presents many advantages $[1,2]$. Such measurements consider the vehicle as a radiating source; hence the FF distance is too large to be obtained in closed areas. Anechoic rooms provide a good solution for small antennas, but for large dimensions, costs become huge.

The proposed method is based on transient metrology [3]; it uses an outdoor time-based measurement base [4]. The system consists of a transient pulse generator connected to the transmitting antenna (antenna to be characterized), and a transient probe as a receiving antenna, connected to a performing data acquisition system (real time scope). This system allows getting field values at the wanted positions by generating a very narrow pulse, which does not disturb the electromagnetic environment, and in which spectral content corresponds to a large frequency spectrum.

The transient outdoor measurement method presents many advantages [5]. In fact, absorbent materials are not efficient for measurements in low-frequency domain. Besides, measuring in frequency domain needs expensive equipments, long time, and different types of absorbent materials for a wideband frequency analysis. The cost of anechoic room increases faster when increasing the antenna dimensions. These problems are solved using outdoor transient measurement base. Simple installation and unlimited dimensions decrease the measurement costs. The generated time pulse permits to get values at any frequency in the spectrum covered by the signal, using time windowing and appropriate Fourier Transform techniques.

In the following, we will characterize an Ultra-Wideband (UWB) antenna in order to validate the proposed method. The principle of the method and the used antenna will be presented in the first part, near field to far field transformation in cylindrical coordinates in second part, in order to explain the experimental setup and results in the last part.

\section{Principle of the Method}

The UWB transient measurement technique in outdoor is well suited to the characterization in near field. In fact, far field ranges of large antenna (as well as a radiating antenna placed on a vehicle) require a huge infrastructure and an important radiated peak power. In such cases, the elimination of the influence of the environment could be difficult. 


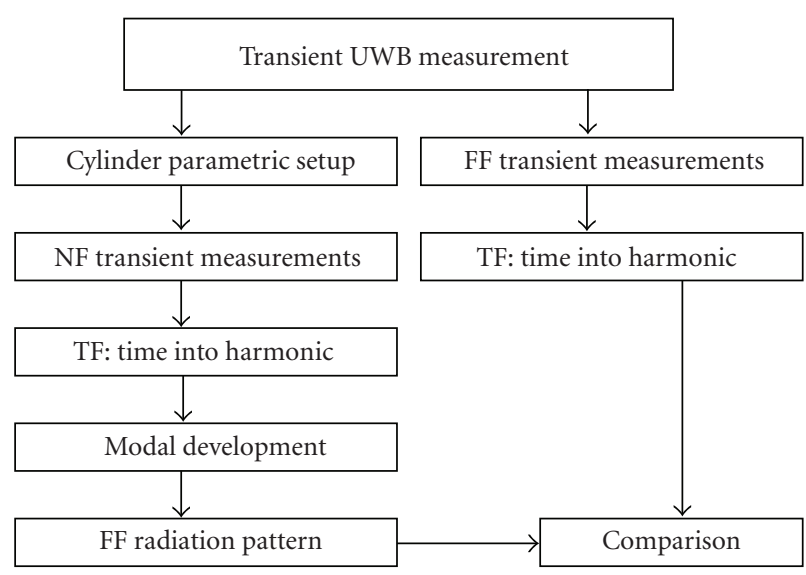

FIGURE 1: Synoptic of the validation.

Starting from near field transient measurements, the fields' calculation for all frequencies is deduced from the Fourier transform, and the construction of the far field can then be made by the application of specific algorithms.

Near Field to Far Field transformations are based on two principles. The first is to measure the values of $\mathrm{E}$ and $\mathrm{H}$ fields on a closed surface (Huygens), and then to seek secondary radiating sources using integral equations. The second is to perform a modal development of the tangential near electric field, as a solution of the vector Helmotz equation of propagation, which verifies the boundary conditions on the measurement surface at Near Field as well as at infinity. The theoretical formulation of this transformation exists in three coordinate systems: planar [6], cylindrical $[1,2,7]$, and spherical [8]. The antenna's nature determines the required geometry.

The modal development method [1,2] permits the construction of the far field's radiation pattern as function of the tangential Electric near field. Cylindrical coordinates system is chosen for simplicity in installations using a vertical mast and a turntable. This method is adapted for any radiating source. The method's validation (Figure 1) necessitates a radiating source with a "known" FF radiation, in order to compare it with what we will construct from the measured NF data.

The radiating source is a transmitting antenna which radiates ultra-short pulses with very low dispersion. The chosen antenna is the UWB SCISSOR antenna (Figure 2) $[4,9]$, directive in both $\mathrm{E}$ and $\mathrm{H}$ planes. This antenna measures $60 * 100 \mathrm{~cm}$.

The scissor antenna is simulated in CST MICROWAVE STUDIO software (Figure 3).

To analyze the radiation of this antenna, we used a transient solver, with a mesh dimensions going to a maximum of $\lambda / 20$. The excitation transient signal feeding the antenna is a Gaussian having a rise time of 280 ps. Its spectrum covers the frequency band $30 \mathrm{MHz}-1.5 \mathrm{GHz}$. The potential difference at the antenna's entry has a value of 7 volts. The simulation results of the FF patterns at a distance of 10 meters for a working frequency of $1 \mathrm{GHz}$ for $\mathrm{E}$ plane

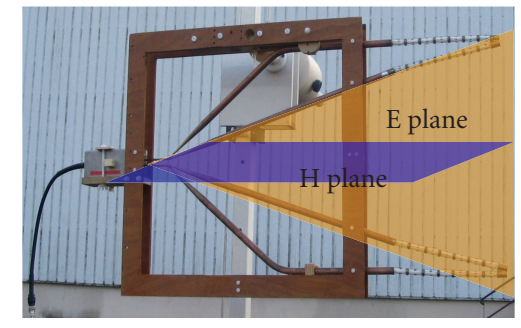

FIgURE 2: Scissor antenna.

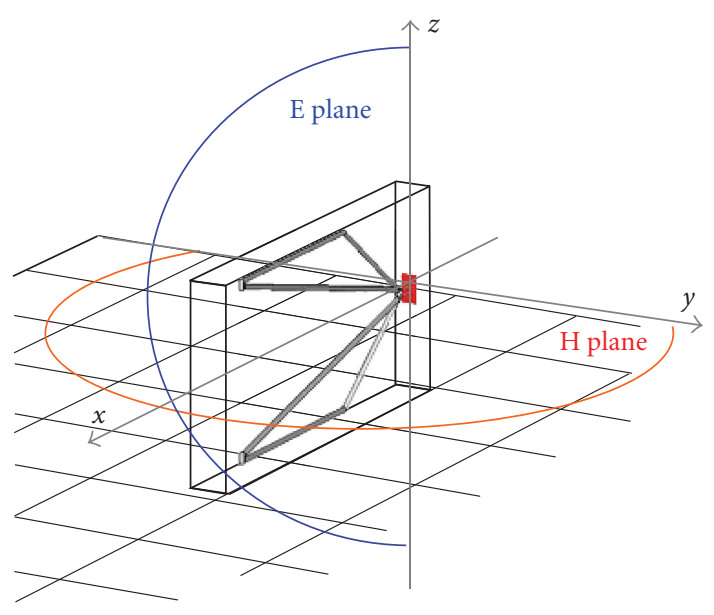

FIgURE 3: Simulated scissor antenna.

and $\mathrm{H}$ plane are shown, respectively, in spherical coordinates system on Figures 4 and 5.

\section{Near Field to Far Field Transformation Technique}

This method deals with the reconstruction of Far-Field $\left({ }^{F}\right.$ index $)$ radiation pattern from Near-Field $\left({ }^{N}\right.$ index $)$ measurements on a cylinder. The tangential NF components lead to FF pattern using an expansion in terms of modes, as a linear combination of elementary solutions of the vector wave equation outside the antenna $[1,2,7,10,11]$.

The measurement cylinder is chosen around the tested antenna (Figure 6), in the NF zone with a radius "a." The tangential components of the electric NF, radiated by the antenna, can be written as a superposition of elementary cylindrical waves:

$$
\begin{aligned}
& E_{z}^{N}(\phi, z)=\sum_{n=-\infty}^{+\infty} \int_{-\infty}^{+\infty} b_{n}\left(k_{z}\right) \frac{\Lambda^{2}}{k} H_{n}^{(2)}(\Lambda a) e^{j n \phi} e^{-j k_{z} z} d k_{z}, \\
& E_{\phi}^{N}(\phi, z) \\
& =\sum_{n=-\infty}^{+\infty} \int_{-\infty}^{+\infty}\left(b_{n}\left(k_{z}\right) \frac{n \cdot k_{z}}{k a} H_{n}^{(2)}(\Lambda a)\right. \\
& \left.\quad-\left.a_{n}\left(k_{z}\right) \frac{\partial H_{n}^{2}(\Lambda r)}{\partial r}\right|_{r=a}\right) e^{j n \phi} e^{-j k_{z} z} d k_{z},
\end{aligned}
$$




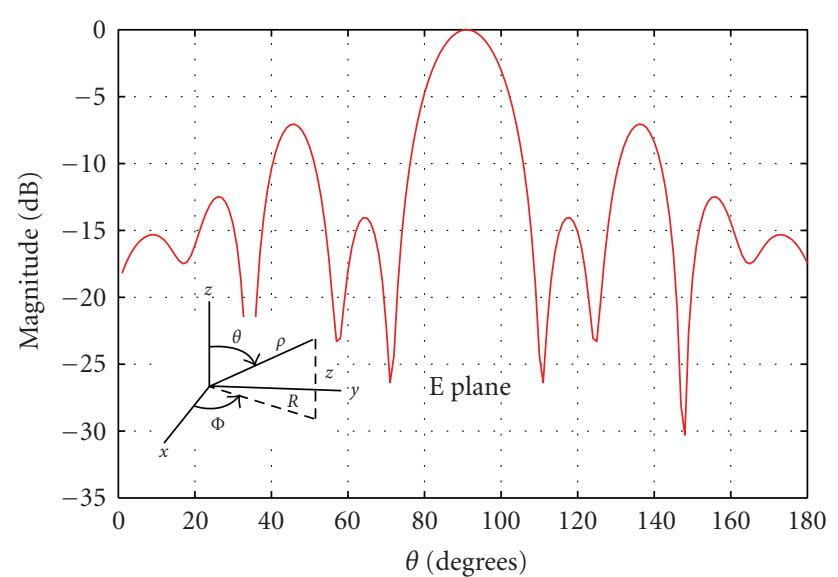

- FF $1 \mathrm{GHz}$

Figure 4: Normalized FF E plane $1 \mathrm{GHz}$.

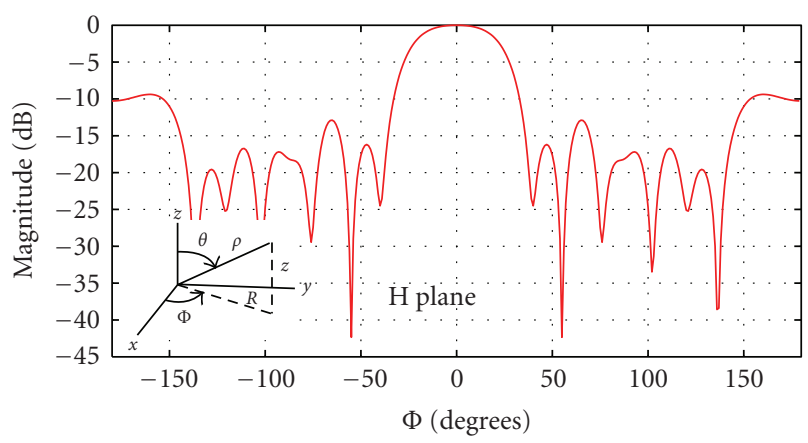

- FF $1 \mathrm{GHz}$

Figure 5: Normalized FF H plane $1 \mathrm{GHz}$.

where " $a$ " is the NF measurement cylinder radius, $k=2 \pi / \lambda$ is the wave vector, and " $n$ " is the mode number.

$H_{n}^{(2)}(\Lambda a)$ is the Hankel function of second kind, of order $n$, with $\Lambda=\sqrt{k^{2}-k_{z}{ }^{2}}$.

From these equations, we can get the modal coefficients $a_{n}\left(k_{z}\right)$ and $b_{n}\left(k_{z}\right)$, as functions of two-dimension spatial integrals of $E_{z}^{N}(\phi, z)$ and $E_{\phi}^{N}(\phi, z)$ :

$$
\begin{aligned}
b_{n}\left(k_{z}\right)= & \frac{k}{\Lambda^{2 H_{n}^{(2)}}(\Lambda a)} \varepsilon_{v}\left(n, k_{z}\right), \\
a_{n}\left(k_{z}\right)= & \frac{1}{\partial H_{n}^{(2)}(\Lambda r) /\left.\partial r\right|_{r=a}} \\
& \times\left[b_{n}\left(k_{z}\right) \cdot \frac{n \cdot k_{z}}{k \cdot a} \cdot H_{n}^{(2)}(\Lambda a)-\varepsilon_{H}\left(n, k_{z}\right)\right],
\end{aligned}
$$

where $\varepsilon_{H}\left(n, k_{z}\right)$ and $\varepsilon_{v}\left(n, k_{z}\right)$ are the two-dimensional transformation of the measured NF according to $\Phi$ and $z$ directions, respectively:

$$
\begin{gathered}
\varepsilon_{H}\left(n, k_{z}\right)=\frac{1}{4 \pi^{2}} \int_{-\infty}^{+\infty} \int_{-\pi}^{+\pi} E_{\phi}^{N}(\phi, z) e^{-j n \phi} e^{+j k_{z} z} d \phi d z, \\
\varepsilon_{v}\left(n, k_{z}\right)=\frac{1}{4 \pi^{2}} \int_{-\infty}^{+\infty} \int_{-\pi}^{+\pi} E_{z}^{N}(\phi, z) e^{-j n \phi} e^{+j k_{z} z} d \phi d z .
\end{gathered}
$$

Using these coefficients, we can reconstruct the FF radiation pattern in spherical coordinates system as follows:

$$
\begin{aligned}
& E_{\theta}^{F}(\theta, \phi)=-j 2 k \sin \theta \cdot \frac{e^{-j k R}}{R} \sum_{n=-\infty}^{+\infty} j^{n} b_{n}(k \cos \theta) e^{j n \phi}, \\
& E_{\phi}^{F}(\theta, \phi)=-2 k \sin \theta \cdot \frac{e^{-j k R}}{R} \sum_{n=-\infty}^{+\infty} j^{n} a_{n}(k \cos \theta) e^{j n \phi},
\end{aligned}
$$

where " $R$ " is the FF distance.

These equations relate the modal coefficients of the tangential fields on the NF measurement cylinder.

So, using the tangential components of the electric field, we can get the modal coefficients of the cylindrical wave expansion $a_{n}\left(k_{z}\right)$ and $b_{n}\left(k_{z}\right)$ by applying a two-dimensional Fast Fourier Transform (FFT_2D).

It is very important that acquisition steps respect some criteria since we deal with Fourier Transform. Sample spacing must be such as to make aliasing error negligible. The standard sampling criteria adopted in cylindrical NF-FF transformation $[10,11]$ are

$$
\Delta z \leq \frac{\lambda}{2}, \quad \Delta \phi \leq \frac{\pi}{k \rho}=\frac{\lambda}{(2 \rho)},
$$

where " $\rho$ " is the radius of the smallest sphere enclosing the antenna under test. The first condition fixes the height of each sample $\Delta z$, and the second fixes an angular increment $\Delta \phi$. It is interesting to measure on a cylinder having the smallest radius in the NF region, in order to minimize the number of acquired points.

\section{Experimental Setup and Results}

For the scissor antenna we used a shadow cylinder of radius $110 \mathrm{~cm}$, centered at the antenna's feeding point (Figure 6). Respecting the sample spacing criteria (5) for the working frequencies $1 \mathrm{GHz}$ and $600 \mathrm{MHz}$, we will use a step of $10 \mathrm{~cm}$ for height and 10 degrees for angles.

The radiating source (scissor antenna) will be connected to a transient pulse generator through a matching balun. The generated pulse feeding the antenna is a Gaussian having a rise time of $116 \mathrm{ps}$ (Figure 7). Its spectrum covers the frequency band $30 \mathrm{MHz}-1.5 \mathrm{GHz}(-3 \mathrm{~dB})$ (Figure 8).

The used probe is a transient electric field sensor AD- 80 model (Figure 9) made by Prodyn. The output of this sensor is a potential $V$, from which we can get the measured electric field as follows [12]: 


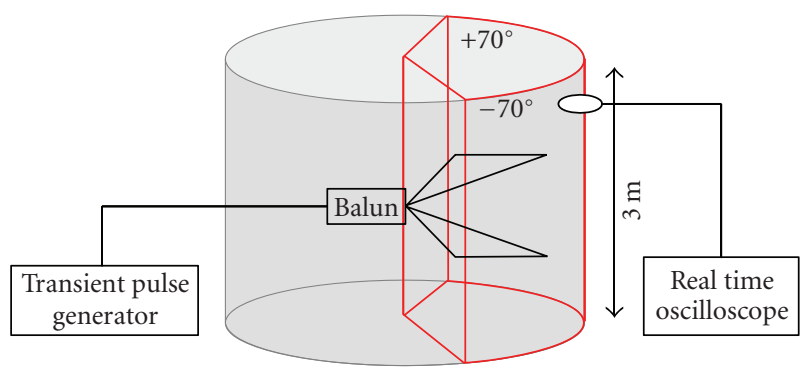

FIgURE 6: Experimental setup.

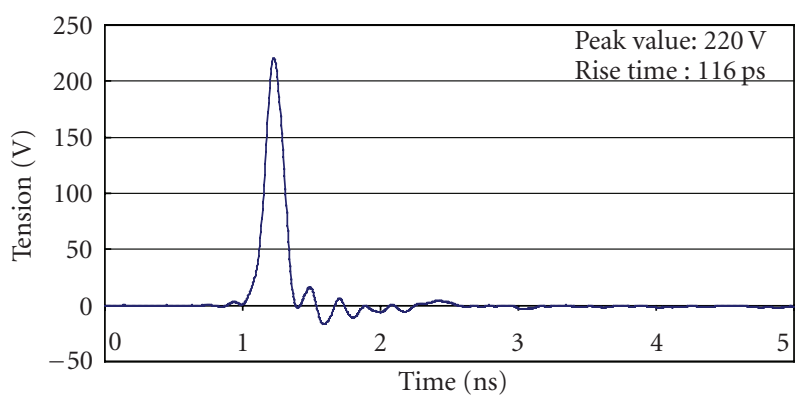

FIgURE 7: Generated time pulse.

$$
E(t)=\frac{1}{R \cdot A_{\mathrm{eq}} \cdot \varepsilon 0} \cdot \int_{0}^{t} V(u) d u,
$$

where $V$ is the output voltage of the sensor, $R$ is the impedance characteristic (100 ohms), $A_{\mathrm{eq}}$ is the sensor equivalent surface of $3 \times 10^{-4} \mathrm{~m}^{2}$, and $\varepsilon_{0}$ is the free space permittivity, cutoff frequency $5.5 \mathrm{GHz}$.

The sensor must be oriented in a way so that antenna's radiations arrive orthogonal to the ground plane of the sensor.

In the following, we will measure only one component of electric NF, $E_{z}$; so we will be able to construct only the $E_{\theta}$ component in the FF.

The measured electric near field is a signal of two parts, one containing the energy of the radiating pulse, and the other is the reflections from the surrounding to be eliminated. Time windowing is a useful operation to be done on this stage (Figure 10). It takes into account the propagation time of reflections from ground, walls, and other existing obstacles.

By applying Fourier Transform, we can get the field values at $1 \mathrm{GHz}$ and $600 \mathrm{MHz}$; then applying the appropriate algorithms of the modal development method, we can construct the far field of this antenna.

Another measurement is done, for $\mathrm{E}$ and $\mathrm{H}$ planes, directly in far field. It allows comparing with the constructed one. This measurement is done in time domain with the same probe; Fourier transform permits to obtain needed values for each frequency covered by the excitation signal.

The comparison of the constructed FF and the measured one, for a working frequency of $1 \mathrm{GHz}$ and then at $600 \mathrm{MHz}$, is given in Figures 11 to 14 .

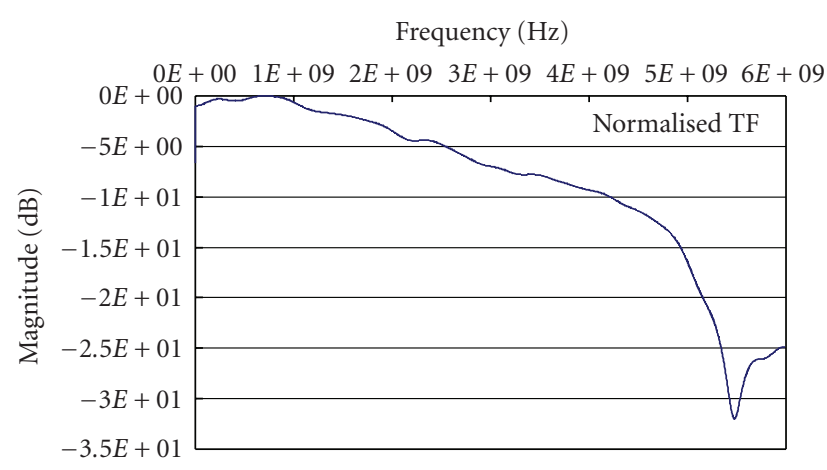

Figure 8: Normalized spectrum.

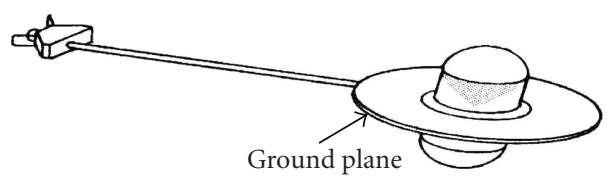

Figure 9: Transient E field sensor.

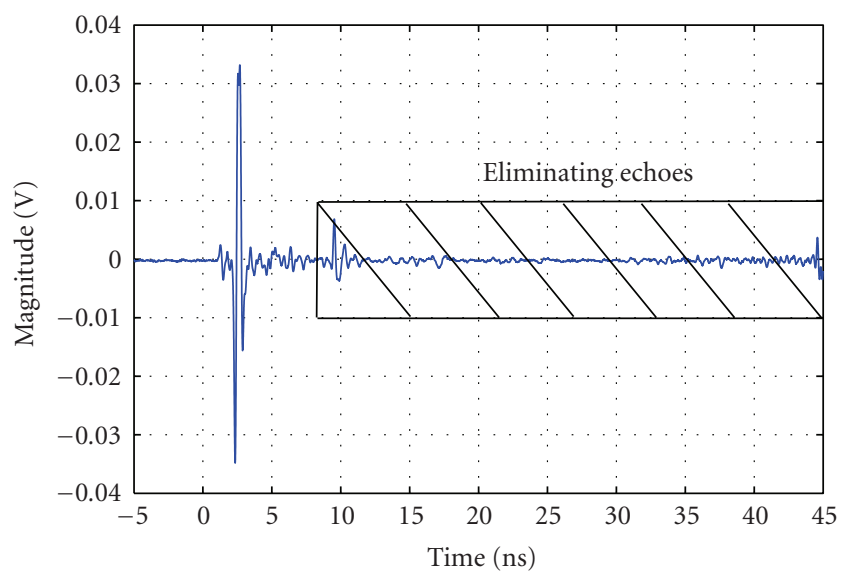

FIgURE 10: Time Windowing.

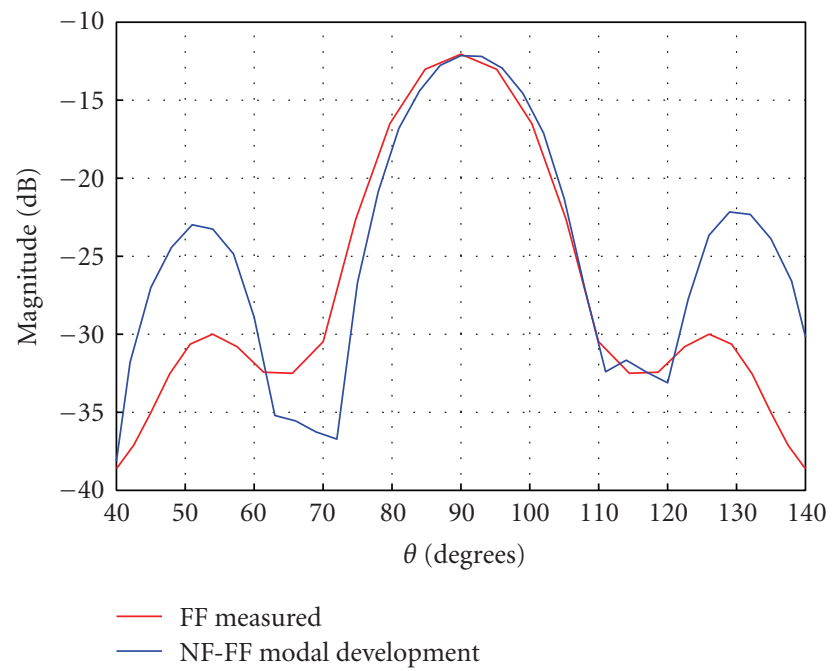

Figure 11: FF $E_{\theta}$ at $1 \mathrm{GHz}$ in E plane. 


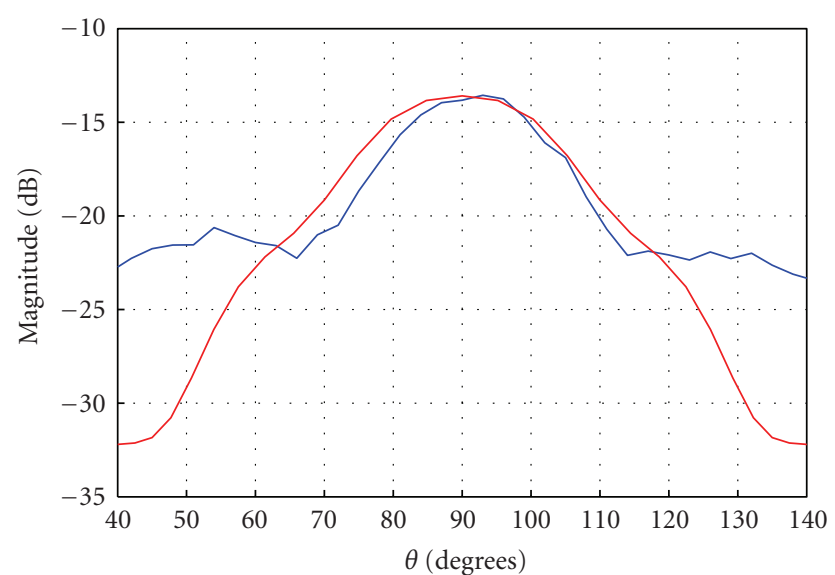

- FF measured

— NF-FF modal development

Figure 12: FF $E_{\theta}$ at $600 \mathrm{MHz}$ in E plane.

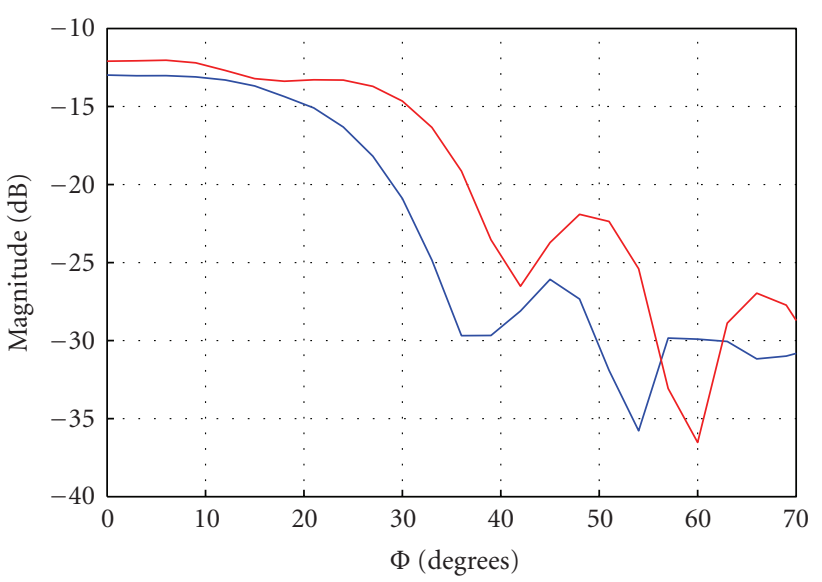

- FF measured

— NF-FF modal development

Figure 13: $\mathrm{FF} E_{\theta}$ at $1 \mathrm{GHz}$ in $\mathrm{H}$ plane.

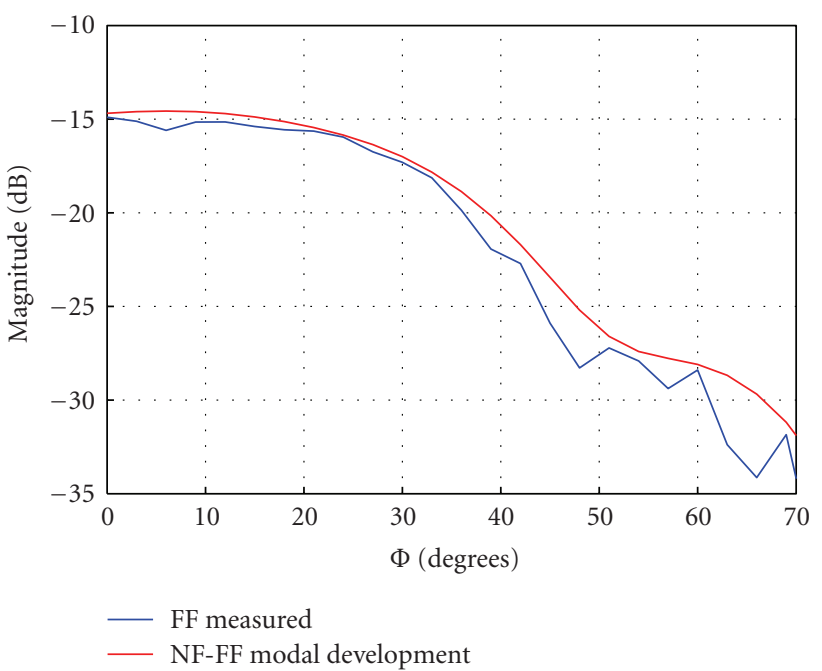

Figure 14: FF $E_{\theta}$ at $600 \mathrm{MHz}$ in $\mathrm{H}$ plane.

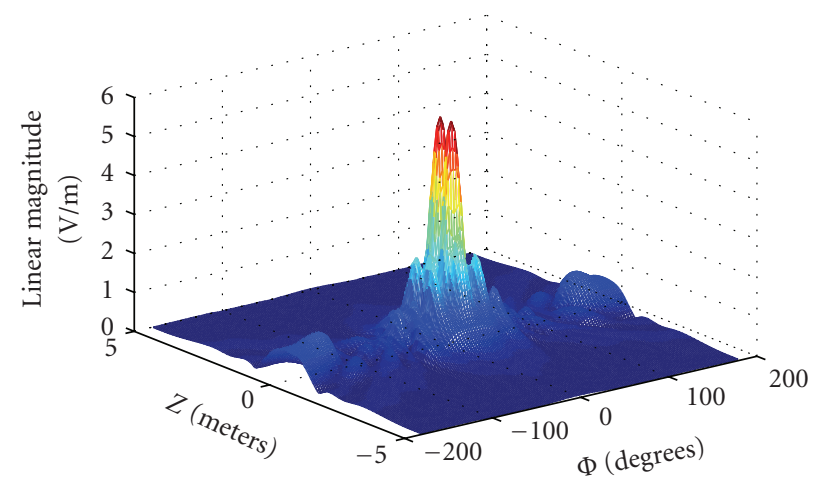

FIGURE 15: Reduced height NF cylinder for $E_{z}$ at $1 \mathrm{GHz}$.

These results show accordance between the FF values calculated from measured NF and the measured FF of the scissor antenna, in two different planes. It should be noted that this measurement is done on a prototype non automated measurement base. The validation of the method can be concluded by these comparisons.

By comparing the different results of Figures 11 and 12 for $E_{\theta}$ in the $E$ plane and Figures 13 and 14 for $E_{\theta}$ in the $\mathrm{H}$ plane, when decreasing the working frequency from $1 \mathrm{GHz}$ to $600 \mathrm{MHz}$, we realize that the diagram shows a difference when the acquired field is not in front of the transmitting antenna $\left(\theta=90^{\circ}, \phi=0\right)$. This is one of the limitations of this NF-FF transformation method in cylindrical form. At low frequencies, the antenna NF radiations are not very directive; therefore they exist over the cylinder with the used height. The ideal case is a cylinder going to infinity.

\section{Influence of NF Data Reduction Simulation}

The FF construction in predefined planes can be achieved with selected NF data on the measurement cylinder.

The condition is to take the entire field spot presented on the cylinder. Figures 15, 16, 17, 18, and 19 explain better this idea.

In height, we are able to construct the FF values by taking measurements up to $z= \pm 1.4 \mathrm{~m}$ (Figure 15).

Influence of Height Truncation. The constructed FF in the E plane for two different heights is shown in Figure 16.

Influence of Angle Truncation. The same idea works in the $\mathrm{H}$ plane. Taking the NF radiations in front of the antenna (Figure 17) allows us to construct the same FF in $\theta$ direction in the E plane facing the antenna (Figure 18), and the part of this field in $\mathrm{H}$ plane going to $\Phi= \pm 90$ degrees in phi (Figure 19).

Figure 19 shows a fall at $\Phi=70^{\circ}$ comparing with the complete FF radiation in this plane. This is due to the angle truncation of NF data on the acquisition cylinder.

These results have been validated for all planes defined by $\theta=$ constant or $\Phi=$ constant. 


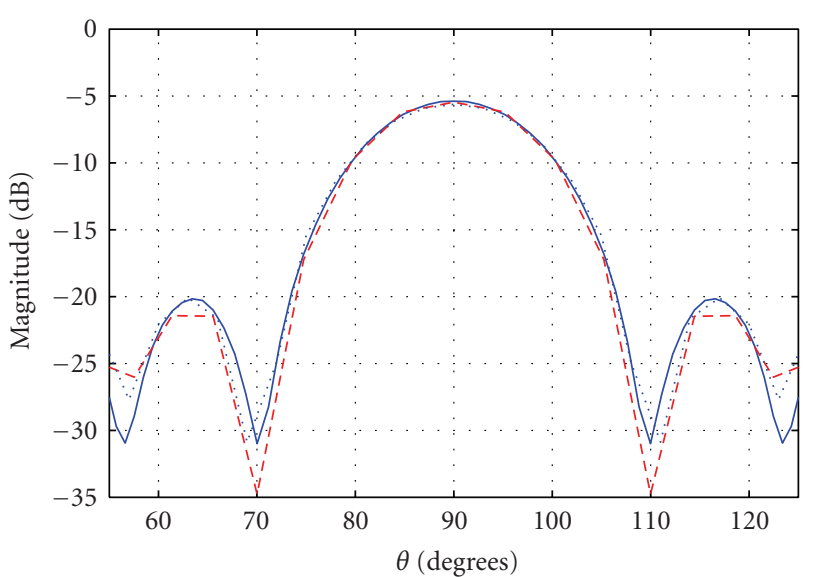

- Modal development from $5 \mathrm{~m}$ height Modal development from $1.4 \mathrm{~m}$ height - - CST

Figure 16: FF $E_{\theta}$ at $1 \mathrm{GHz}$ in E plane.

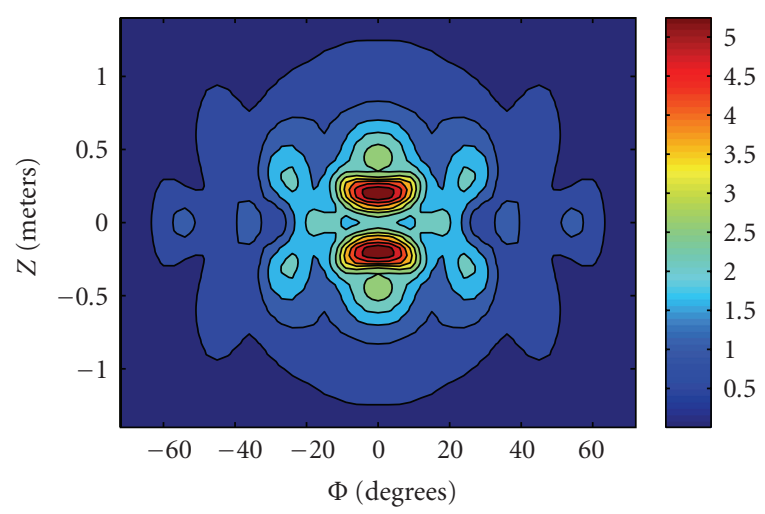

FIgURE 17: Choice of data spot on NF cylinder for $E_{\mathrm{z}}$ at $1 \mathrm{GHz}$.

\section{Conclusion and Perspective}

This measurement base allows the Far Field determination of antennas and radiating sources from a single transient outdoor acquisition. It provides a notable gain in terms of measurement time and costs, while remaining nondisruptive to the environment with pulses of very short durations.

This measurement technique is more efficient in Near Field ranges. Using NF-FF transformation techniques, it allows us to obtain the radiation pattern of any radiating structure over a wide band of frequencies, from a single measurement followed by a mathematical calculation.

The next work will be oriented in two ways. First we will improve the modal development in cylindrical coordinates to get more accurate FF patterns, while the second way will be the determination of FF directly in time domain, without time to frequency conversion [13-16].

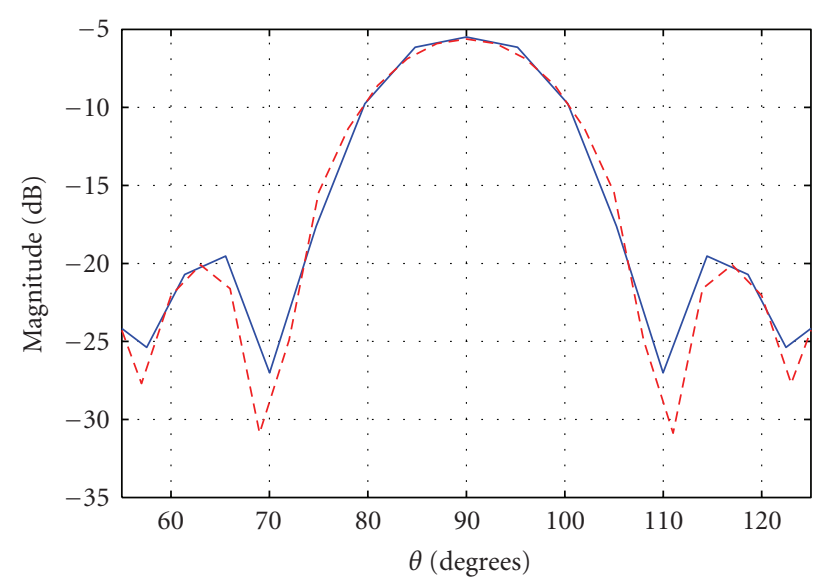

- Modal development

--- CST

Figure 18: FF $E_{\theta}$ at $1 \mathrm{GHz}$ in E plane.

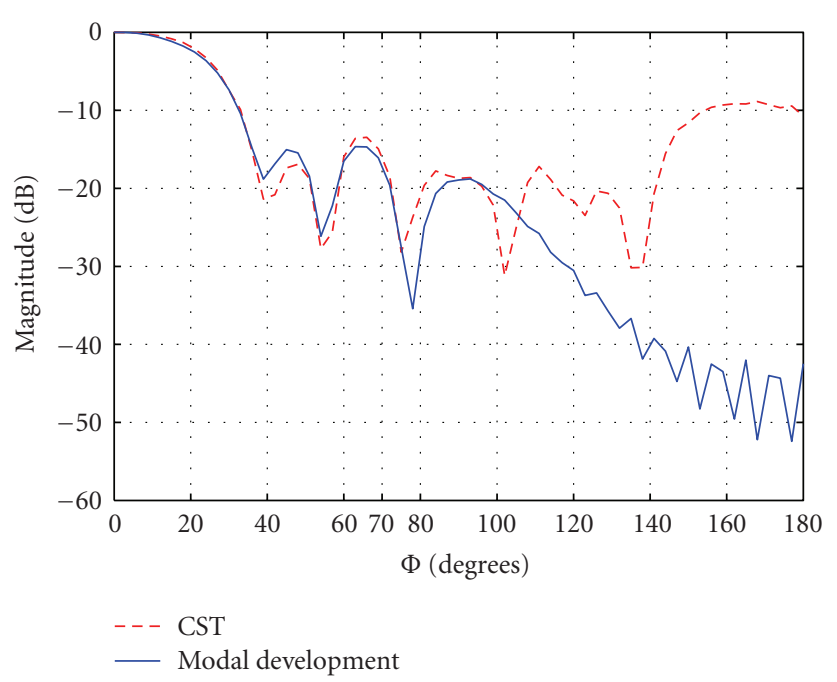

Figure 19: $\mathrm{FF} E_{\theta}$ at $1 \mathrm{GHz}$ in $\mathrm{H}$ plane

\section{References}

[1] W. M. Leach Jr. and D. T. Paris, "Probe compensated near-field measurements on a cylinder," IEEE Transactions on Antennas and Propagation, vol. 21, no. 4, pp. 435-445, 1973.

[2] O. M. Bucci, C. Gennarelli, G. Riccio, and C. Savarese, "NFFF transformation with cylindrical scanning: an effective technique for elongated antennas," IEE Proceedings: Microwaves, Antennas and Propagation, vol. 145, no. 5, pp. 369-374, 1998.

[3] E. Martinod, V. Bertrand, M. Lalande-Guionie, A. Reineix, and B. Jecko, "Behavior of multifilar connectors in electromagnetic compatibility: a new experimental transient approach," IEEE Transactions on Electromagnetic Compatibility, vol. 44, no. 3, pp. 424-433, 2002.

[4] J. Andrieu, S. Nouvet, V. Bertrand, B. Beillard, and B. Jecko, "Transient characterization of a novel ultrawide-band antenna: the scissors antenna," IEEE Transactions on Antennas and Propagation, vol. 53, no. 4, pp. 1254-1261, 2005.

[5] J. Young, D. Svboda, and W. Burnside, "A comparison of timeand frequency-domain measurement techniques in antenna 
theory," IEEE Transactions on Antennas and Propagation, vol. 21, no. 4, pp. 581-583, 1973.

[6] D. M. Kerns and E. S. Dayhoff, "Theory of diffraction in microwave interferometry," Journal of Research of the National Bureau of Standards, vol. 64B, pp. 1-13, 1960.

[7] J. A. Stratton, Electromagnetic Theory, MacGraw Hill, New York, NY, USA, 1941.

[8] P. D. Potter, "Application of spherical wave theory to Cassegrainian-fed paraboloids," IEEE Transactions on Antennas and Propagation, vol. 15, no. 6, pp. 727-736, 1967.

[9] J. Andrieu, B. Beillard, and Y. Imbs, "Wide band scissors antenna," French patent no. 99 14940, November 1999, International patent no. FR 0002905, October 2000.

[10] O. M. Bucci and C. Gennarelli, "Use of sampling expansions in near-field-far-field transformation: the cylindrical case," IEEE Transactions on Antennas and Propagation, vol. 36, no. 6, pp. 830-835, 1988.

[11] O. M. Bucci, C. Gennarelli, G. Riccio, V. Speranza, and C. Savarese, "Efficient near-field-far-field transformation with cylindrical scanning by a finite and non redundant number of data," IEEE Transactions on Antennas and Propagation, vol. 1, no. 1, pp. 256-259, 1995.

[12] http://www.prodyntech.com/ddotff.htm.

[13] T. B. Hansen and A. D. Yaghjian, "Formulation of timedomain planar near-field measurements without probe correction," Tech. Rep., Rome Laboratory, Hanscom AFB, Mass, USA, September 1993.

[14] T. B. Hansen and A. D. Yaghjian, "Planar near-field scanning in the time domain-part 1: formulation," IEEE Transactions on Antennas and Propagation, vol. 42, no. 9, pp. 1280-1291, 1994.

[15] T. B. Hansen and A. D. Yaghjian, "Planar near-field scanning in the time domain-part 2: sampling theorems and computation schemes," IEEE Transactions on Antennas and Propagation, vol. 42, no. 9, pp. 1292-1300, 1994.

[16] T. B. Hansen and A. D. Yaghiian, "Formulation of probecorrected planar near-field scanning in the time domain," IEEE Transactions on Antennas and Propagation, vol. 43, no. 6, pp. 569-584, 1995. 

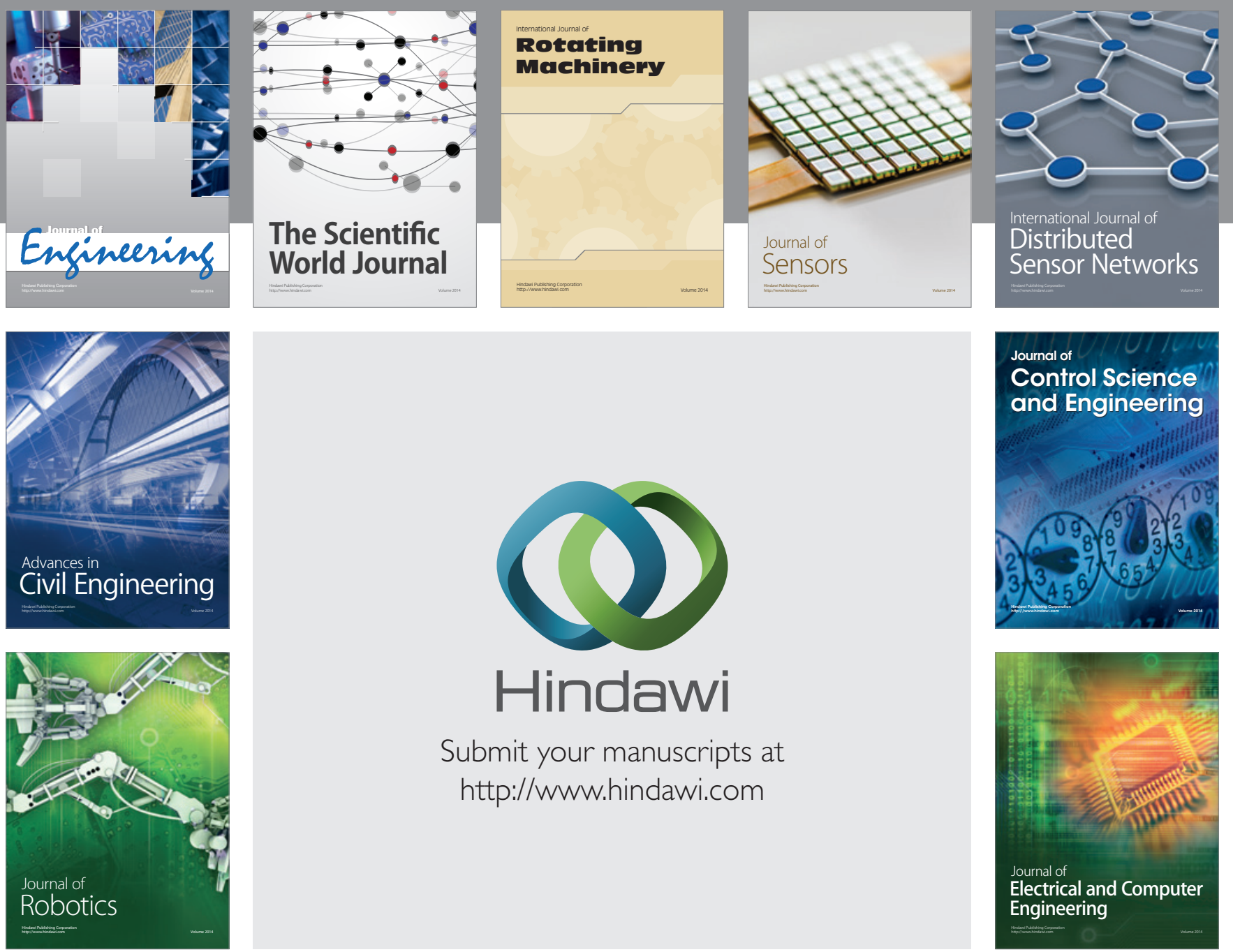

Submit your manuscripts at

http://www.hindawi.com
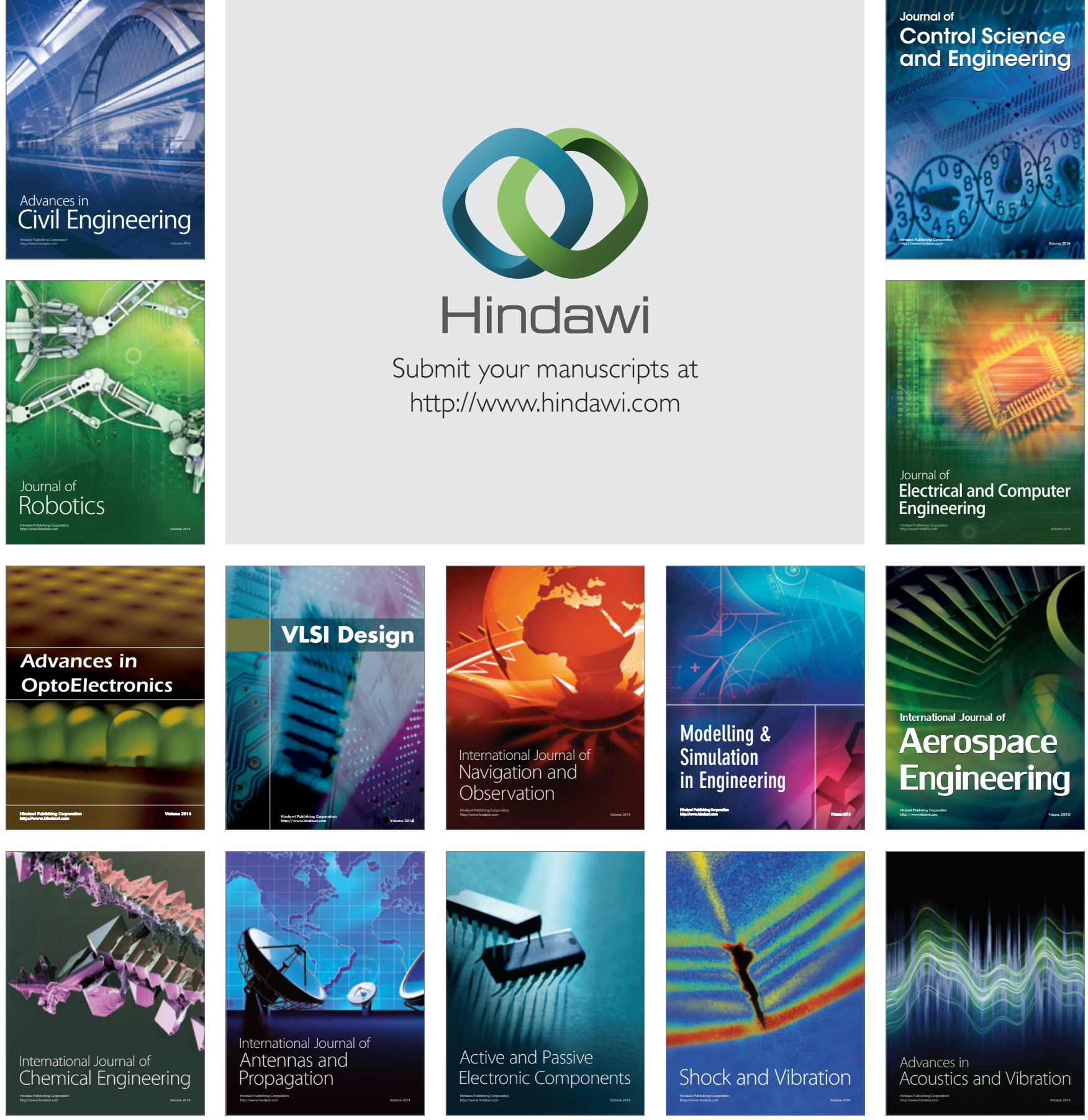